\title{
Enteroparasitosis en jardines infantiles de Vallenar
}

\author{
Dr. Roberto Figueroa \$.; Matr. Luz A. Valenzuela G. ${ }^{2}$ T.M. Edecia Flores B. ${ }^{3}$ \\ Intestinal parasitosis among children attending day \\ care centers and their care takers
}

\begin{abstract}
A prospective study was hejd on august and september (late winter and early spring) of 1985 among children ant their adult care takers irom day care centers at Vallenar, Chile: three stool and one Graham's tape test samples were taken from 534 subjects (485 children). Evidence of intestunal parasitosis was detected in $281 / 534$ (52.6\%) of the study population (362/485 or $54.2 \%$ of children and 18,49 or $36.7 \%$ of adults). The most frequently isolated intestinal parasites were Giardia lamblia and Enterobius vermicularis (40.2 and $10.3 \%$ of subjects respectively). Several parasites were simaltaneously detected from the same samples of $103 / 281$ (36.7\%) of the infected people. Intestinal parasites were mostly detected from patients of lower socioeconomic status (75.4\%of those with evidence of infection came from levels 1 and 2 of a 5 point scale).
\end{abstract}

(Key words: intestinal parasites, chíldren, care takers, day care centers).

Uno de los problemas de mayor jerarquia en salud pública, lo constituyen las enteroparasitosis, como lo reflejan los múltiples y variados trabajos sobre ellos. ${ }^{1-4}$ Desgraciadamente, esta lacra compromete con mayor frecuencia a nuestros hijos, patrimonio potencial en pleno crecimiento $\mathrm{y}$ desarrallo; creando alteraciones de diversa indole en ellos. ${ }^{2.5,6}$ Además, la existencia de parasitos intestinales tiene mayor magnitud en aquellos sitios que concentran niños supuestamente sanos, como los jardines infant1les. 1.4-6

Partiendo de estas tres premisas, se explica el interés que nos motiva, de establecer un diagnóstico de esta situación en nuestra ciudad; toda vez que ella beneficiara a la muestra en estudio, la comunidad en general, servirá de información adicional para futuras investigaciones $y$, además brindará un valioso complemento actualizado que redundará en una mejoría de los indices de salud en nuestra suciedad.

\section{MATERIAL Y METODOS}

Se estudió en formu prospetiva, entre los mests de agosta $y$ septicmbre de 1985, una muestra representativa de niños $y$ adultos de tres jardines infantiles, dos del sector privado y uno de la municipalidad, en Vallenar. Fodos en su conjunto atjenden a una población bastante heteroginea en nivel socio-económico

Se adaptó una indagatoria tipo, original de los jutores, basada en la concuesta de listratificación Social,

1. Médico pediatra

2. Matrona

3. Tecnókgo Médico, Vallunár. vigente en todo el país, aplicada por los respectivos municipios. Está ajustada a los siguientes indicadores: indice cuma por persona, situación laboral y años de estudio del jefe de hogar, raguá potable, luz eléctrica, aicantarillado intradomiciliario, además de la recolección de basuras) e ingreso mensual del hogar expresado en dólares de EE.LU. calculados mediante el valor promedio oficial de scptiembre de 1985 de la moneda chilena ( $\$ 180 \times$ US\$).

La aplicación de esta encuestra dio origen a cinco grupos de indices-tipos:

1: jefe de hogar sin trabajo estable o adscrito al Plan de empleo mínimo (PEM), indice camasipersona menor de 0,6 ; dos o menos parámetros de saneamiento búsico; escolaridad menor de 4 años, e ingreso mensual menor de L'S\$ 39. II: jete de hogar en el Plan de absorción de mano de obra para jefes de hogar (POJH). indice camas/personas entre 0.6 y 0.69 ; dos o tres parámetros de saneamiento básico; escolaridad entre 4 y 7 añus; e ingreso mensual entre US\$ 40 y 59 . III: jefe de hogar obrero estable: indice camas/personas entre 0.7 y 0,79 ; sancamiento básico completo; escolaridad de 8 a 11 años; e ingreso mensual entre US\$ 60 y US\$ 139. IV: jefe de hogar empleado: índice camasipersonas intre 0,8 y 0,89 ; saneamiento básico completo: escola. ridad de 12 a 16 años: íngreso mensual entre LSS 140 y US\$249. V: jefe de hogar profesional, indice camasipersonas mayor de 0.9 ; saneamicnto basico conpleto: cscolaridad mayor de 16 años; e ingeșo mensual superior a USS 250.

Los parámetros más ficles para catalogar is un grupos familiar en un determinado indice fueron: el ingreso mensual $y$ cl indice cama; en consecuencia, si existía alguлa duda se aplicaba esto último definiendo el indice final Jel grupo en cuestion.

Se efectuó educación sobrc el temu, al personal $y$ apoderados de cada uno de los jardines infantiles. recalcando los objetivos del presente trabajo, la epide miologia, profilaxis y control de las parasitosis, al igual que la toma correcta de las muestras. 6

Se capacitó a algu nas auxiliares y apoderados para la tecolección de los exámenes en el propio jardín infantil. Se instruyó a aquellos padres de ayyos nijos no $x$ 
obtuvo muestra de heces en este sitio, para hacerlo en sus casas.

Los adultos se tomaron el examen en sus hogares, at igual quc la prueba, esta última según modificación propuesta. 7

Se logró recolectas exámenes coproparasitológicos seriados de 3 muestras e igual número de pruebas de Graham, de 534 sujetos ( 495 niños y 49 adultos). Todos los casos con parasitosis, fueron derivados al Centro de Salud correspondiente para el tratamiento respectivo.

\section{RESULTADOS}

La tabla 1 resume la distribución de la muestra, según grupo etario e indice socio-económico. Se evidencia que existe bastante homogeneidad en la distribución ya anotada, en los respectivos parámetros.

La tabla 2 revela la distribución según grupo etario e indice socio-conómico de los parasitados. Llama la atención la presencia de enteroparasitosis en la población de menores recursos, índices I y Il, hecho más claro aún en la tabla 3. Al descomponer esta información según la existencia de mono y poliparasitismo, $178(33,3 \%)$ encuestados tenian monoparasitismo y. 103 $(19,3 \%)$ poliparasitismo, afectándose en ambas situaciones, el grupo de menores ingresos.

Giardia lamblia fue el parásito más frecuentemente aislado en nuestros jardines infantiles, en $40,2 \%$ de los sujetos parasitados; Enterobius yermiculares ocupa el segundo lugar, en $10,3 \%$ de los mismo. La asociación de ambos parásitos estuvo presente en $5,1 \%$ de la muestra, siendo ésta la más frecuente de aquellas.

\section{DISCUSION}

Las enteroparasitosis en los países en vias de desarrollo presentan alta incidencia. ${ }^{3}$ Esto puede ser explicado por saneamiento básico insuficiente, ${ }^{1,4}$ bajo nivel cultural de la población, 5 existencia de vectores, ${ }^{9}$ deficientes prácticas de higiene, ${ }^{3}$ defectuosa manipulación de los alimentos, ${ }^{9}$ hacinamiento, ${ }^{8}$ todo lo cual conlleva marcado fecalismo en la población. ${ }^{10}$

Nuestros resultados apuntan en igual dirección: $52,7 \%$ de parasitados, con $65,6 \%$ de afección en el índice $\mathrm{I}$, disminuyendo progresivamente hasta alcanzar sólo $18,7 \%$ en el indice $V$ (tabla 3).

El hallazgo de Giardia lamblia en forma predominante en jos jardines infantiles ya ha sido comunicado $1,5,6,9$ con cifras parecidas a las nuestras $(40,2 \%)$, lo que contrasta con lo descrito en los paises desarrollados con este parásito y en iguales sitios $(3 \%){ }^{4}$

El poliparasitismo ya ha sido señalado. ${ }^{1} \mathrm{La}$ importancia de nuestras cifras habla en favor de
Tabla 1.

Distribución de 534 pacientes investigados según grupos etáreos e índice socio-económico

\begin{tabular}{lrrrrrr}
\hline $\begin{array}{l}\text { Grupo } \\
\text { de edades }\end{array}$ & \multicolumn{6}{c}{ Indice socio-económico } \\
\hline $\begin{array}{l}\text { Lanctantes } \\
\text { S1 año }\end{array}$ & II & III & IV & V & Muestra \\
Lactantes & & - & 3 & - &.- & 5 \\
>1 año & 7 & 9 & 3 & - & 2 & 21 \\
Prescolares & 105 & 189 & 75 & 8 & 79 & 456 \\
Escolares & - &. & 2 &.. & 1 & 3 \\
Adultos & 8 & 13 & 15 & 4 & 9 & 49 \\
\hline \hline Total & & & & & & 534
\end{tabular}

Tabla 2.

Entcroparasitosis detectadas según grupo etario e indice socio-conómico

\begin{tabular}{|c|c|c|c|c|c|c|}
\hline \multirow{2}{*}{$\begin{array}{l}\text { Grupo } \\
\text { ctarío }\end{array}$} & \multicolumn{5}{|c|}{ lndice sacio-económico } & \multirow[b]{2}{*}{ Total } \\
\hline & I & Il & III & $\Gamma$ & $v$ & \\
\hline $\begin{array}{l}\text { Lactante } \\
\text { menor }\end{array}$ & & & 1 & & & 1 \\
\hline $\begin{array}{l}\text { Lactante } \\
\text { mayor }\end{array}$ & 3 & 6 & 1 & & 1 & 11 \\
\hline Preescular & 74 & 121 & 39 & 2 & 13 & 249 \\
\hline Escolar & & & 2 & & & 2 \\
\hline Adulto & 3 & 5 & 5 & 2 & 3 & 18 \\
\hline Total & 80 & 132 & 48 & 4 & 17 & 281 \\
\hline
\end{tabular}

Tabla 3 .

Distribución de los parasitados con respecto al total de los encuestados según indice socioeconómico

\begin{tabular}{cccc}
\hline Indice & lincuestados & Parasitados & $\%$ \\
\hline V & 91 & 17 & 18,7 \\
IV & 12 & 4 & 33,3 \\
III & 98 & 51 & 52,0 \\
II & 211 & 129 & 61,1 \\
I & 122 & 80 & 65,6 \\
\hline \hline Total & 534 & 281 & 52,6
\end{tabular}

la alta contagiosidad de la giardiasis, ${ }^{9}$ adquiriendo, segun algunos, ${ }^{3}$ cierto carácter familiar. Destaca en esta afección un polimorfismo ${ }^{4,8}$ que contribuye a producir desmedro en el crecimiento $y$ desarrollo del pequefio. ${ }^{6,8}$

Respecto a la oxy.uriasis, nuestras cifras $(10,3 \%)$ son similares a las encontradas por otros autores. ${ }^{1}$ Sólo en esta afección se efectuó el 
Tabl 4.

Distribución de las en teroparasitosis según frecuencia relativa en 281 sujetos infectados

\begin{tabular}{lrr}
\hline Parásitos* & Afectados & $\%$ \\
\hline Giardia lamblia & 113 & $\mathbf{4 0 , 2}$ \\
Enterobius vermiculares & 29 & 10,3 \\
Lndolimax nana & 15 & 5,3 \\
Entamocba coli & 14 & 5.0 \\
Chilomastix maesnilli & 4 & 1,4 \\
Hymenolepsis nana & 3 & 1,1 \\
Asociaciones & 103 & 36,7 \\
\hline Total & 281 & 100,0 \\
* monoparasitisno & &
\end{tabular}

tratamiento familiar en conjunto con el caso indice.

Himenolepiasis se encontró en un $7.8 \%$ de los parasitados, semejante a lo ya comunicado ${ }^{8,9}$ por otros autores.

Tricocefalosis. sólo se encontró en un niño, portador además, de una oxyuriasis. constituyendo su hallazgo una sorpresa en una región como la nuestra.

Siendo los jardines infantiles un recurso indispensable en nuestra sociedad actual,$^{1,6}$ implican riesgo adicional de contaminación a traves de los manipuladores de alimentos, ${ }^{2+10}$

En consideración a estos resultados y a las relaciones que existen entre enteroparasitosis y tallas de sancamiento ambiental de la comuni$\mathrm{dad}^{3,3-5,8-10}$ parece conveniente reforzar las medidas destinadas a mejorar este último $\mathrm{y}$ a exigir at los nitios $y$ a los manipuladores de alimentos. un informe de examen parasitológico seriado de depusiciones y prueba de Graham. con una antigüedad máxima de 30 días, en el momento de incorporarse a los jardines infantiles.

\section{RESUMEN}

Se realizó una investigación prospectiva entre los meses de agosto y septiembre de 1985, de cinco jardines infantiles de Vallenar. Este estudio comprendió la toma de muestras de deposiciones del personal y niños asistentes a aquellos. Fueron estudiadas un total de 534 personas encontrándose un $40,2 \%$ de giardiasis en la población afectada; $10,3 \%$ de oxyuriasis $y$, en $5,1 \%$ la asociación de ambos. Todas las parasitosis encontradas tuvieron mayor incidencia en el grupo más desposeído socio-económicamente. Se discuten algunas normas para su control, sobre todo en los jardines infantiles.

\section{AGRADECIMIENTOS}

Agradecemos al personal profesional y auxiliar de los jardines infantiles encrestados, pot su colaboración: asimismo, a USV Laboratorios S.A., por la donación de su producto Deprocid( $(8)$ en cantidad suficiente para tratar a todos aquellos pacientes que resultaron afectados por alguna protozoosis.

\section{REFERENCIAS}

1. Gottlieb B., Reyes H., Trivino X., Vega J., Arrigga. do L., Hernández J.: Fnteroparasitosis en niños de Jardines lufantiles. Estudio comparativo según nivel socio-económico. Rev Méd Chile 111: 1035, 1983.

2. Campos R., de Souza F.L.: Giardiasis. Invest Méd Internat 10: Supl (5): 50, 1983.

3. Guerra J.G., Tello R., Llanos A., Legua P.: Gjardiasis, Epidemiología, diagnóstico y tratamiento con Tínidazol en Limaì, Perú. Invest Méd Internac 10: Supl (5): $58,1983$.

4. Tumer J.A.: Giamdiasis and Infection with Dientamoeba fragilis. Pediats Clin North An 32: $865,1985$.

5. Noemi l., Reves H., Mauro G.: Infecciones por cnteroparásitos en niños de Salus Cunas y Jardines Intantiles de 2 Hospitales de Sartiago. Rev Chil Pediatr 46: 50, 1975.

6. Gottlieb B., Aguilero X., Reyes H. el al: Enternparasitosis en Jardines Infantiles. Fyaluación de algunas normas de control. Parasitología al día 8: 36 , 1984.

7. Sagkua H. Poblete H.: Pesquisa de infeción por Enterobàs vermictularis en niños de dos internados de la ciudad de Antofagasta. Comparación del método de Grahan con una modificación del mismo. Bol Chil Patasitol 3]: 6, 1976.

8. Fanta E: Parasitosis en el niño. En: Atías, A y Neghme, A. eds: Parasitología Clínica, $2^{2}$ Ed. Mediterráneo, Santiago-Chile, 1984, pay. 94-98.

9. Ramirez R. Schenone H. Galdames M. et al: Frecuencia en Chile de las infecciones humanas por protozoos y helmintos intestinales (1962-1972). Bol Chile Parasitol 27: 116, 1972.

10. Diaz M.C. Muñoz V., Durtuty d. et al: Lstudio bacteriologico y parasitologico en manipuladores de alimentos de servicios hospitalarios. Rev Méd Chile 108: 900,1980 . 